\title{
Medical Science and Physics
}

\author{
Min Raj Lamsal \\ Department of Physics, Prithvi Narayan Campus, Pokhara \\ min_lamsal@yahoo.com
}

\begin{abstract}
The study of physics seems to be theoretical or abstract. The connections between physics and real life can seem remote. But in reality physics is not a purely abstract subject. Like other branches of science, physics has a pure theoretical side and an applied side. The principles of physics are applied in a vast range of contexts from the building of bridges to the design of integrated circuits. Much of the technological revolution has its foundations in the applied physics. One of the major applications of physics which covers vast area of scientific knowledge is medicine. The knowledge of physics is essential for ultrasound, hologram, X-rays, laser therapy, radiotherapy, endoscopy, MRI, CT scanning, ECG, EEG, PET and so on in the field of medical science. The theoretical principles have to be learned and understood first if the applications are to be understood.
\end{abstract}

Keywords: Ultrasound, X-rays, Lasers, Electrocardiogram (ECG), Electro Encephalogram (EEG), Magnetic Resonance Imaging (MRI), Computed tomography (CT) scanning, Positron Emission Tomography (PET)

\section{INTRODUCTION}

Physics is the foundation of not only science but also the society. The development of any country depends upon the development of physics. Physics is the study of matter, energy and their interactions which play a key role in the future progress of humankind. Physics aims to an understanding of world both by observations and of the way in which object behaves offer challenging, exciting and productive career opportunities that cover many specialized fields from medical physics, acoustics, astronomy, astrophysics, geophysics, biophysics, physical chemistry and so on.

Physics is crucial in understanding the world around, inside and the world beyond us. Physics challenges our imagination, and physics based technological developments have revolutionized our world. The knowledge of physics is the foremost need of present day. With lots of inventions and discoveries the world has become a tiny place to live. Human kind has reached to a realm of nature through researches and theories in physics sprouted in the last few centuries. Physics inspires the scientific people to fulfill the needs of the scientific community, in this period of quantum age. Such creation would be memorable and inspirational one for all of us to commit for its improvement in the future. Physics is being used increasingly in all walks of life.

A comprehensive coverage of the role of Physics in the Medical Science would be impossible in an article of this size. So, only an overview of selected techniques which are more frequently used during diagnosis and treatment of different diseases are discussed in brief as given below.

\section{ULTRASOUND:}

Ultrasound can be used to obtain the diagnostic information about the internal structure. Ultrasound obeys the same laws of reflection and refraction as audible sound and light waves. When an ultrasonic wave meets a boundary between two media, some of the wave energy is reflected and some is refracted. Energy considerations make it possible to state that the input intensity $\mathrm{I}$ is equal to the sum of the reflected and refracted intensities $I_{1}$ and $I_{2}$ respectively.

$$
\text { i.e. } I=I_{1}+I_{2}
$$

The relative magnitudes of the reflected and refracted intensities depend on a property of each of the media known as the specific acoustic impedance $Z$. This is the ratio of acoustic pressure to particle velocity but may be taken to be approximately equal to the product of the medium's density and the speed, through the medium, of the wave.

If the ultrasonic waves were to try to pass from one medium into another of much larger impedance, the waves would have difficulty in making the molecules in the denser material vibrate, so most of the wave energy would be reflected and little would be transmitted. Alternatively, if the two media were of similar impedance, the majority of the wave 
energy would be transmitted with greatly differing impedances are human tissue and bone.

$$
Z_{\text {tissue }} / Z_{\text {bone }}=1 / 4
$$

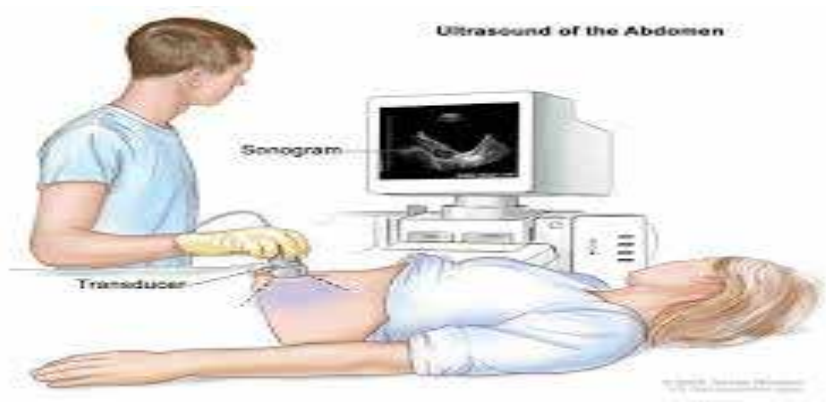

Fig(i) Ultrasound

Ultrasonic waves striking a tissue - bone boundary from the tissue would be mainly reflected. However, tissue and water have similar impedances, so most ultrasonic waves would be transmitted straight through a tissue-water boundary.

Another factor which affects the intensity of ultrasonic waves passing through a medium is absorption. As the waves travel through a medium, the intensity falls exponentially, because energy is absorbed by the medium. This causes the temperature of the medium to increase. The intensity $\mathrm{I}$ at a particular point is given by

$$
\mathrm{I}=\mathrm{I}_{0} \mathrm{e}^{-\mathrm{kx}} \text {, }
$$

where $\mathrm{x}$ is the distance travelled in the medium by the wave, $\mathrm{I}_{0}$ is the original intensity and $\mathrm{k}$ is an absorption constant.

Diagnostic information about internal structure of the body can be obtained by monitoring the pulses reflected back when short bursts of ultrasound have been transmitted into the body. Two techniques, A scan and B scan, are in common use. However, initially, there is a major problem in actually transmitting ultrasound into the human body. This is because the impedances of the air and the skin are vastly different and this causes the majority of ultrasound wave energy incident on the air-skin boundary to be reflected without entering the body. One method originally used to overcome this problem is to immerse a portion of the body and the transducer in water. Then, since the impedance of water and skin are similar, reflection is minimal and most of the wave energy is transmitted. Nowadays, a material called a coupling medium is inserted between ultrasonic transducer and skin to reduce the size of the impedance change between boundaries. At present, a film of oil or water- based cellulose jelly is used as a coupling medium for general use.

The A-scan system basically measures the distance of boundaries from the transducer, with the transducer kept in one position. A short burst of ultrasound is transmitted to the body through the coupling medium. At each boundary between different media in the body, some ultrasound is reflected and this reflected pulse is picked up by the transducer which now acts as a receiver. The signal from the transducer is amplified and displayed on a cathoderay oscilloscope (CRO). The reflected ultrasound also meets boundaries on its return trip to the transducer, causing some energy to be reflected and some transmitted. As one consequence of this, echoes from boundaries deeper in the body tend to be weaker. To compensate for this loss in energy, the later the echo is received at the transducer, the more it is amplified before display on the CRO. A line appears on the CRO screen each time an echo returns to the receiver. The time-base on X-plates is adjusted so that a complete picture of the reflections is received on one scan. Then, by knowing the speed of transmission of the ultrasound, this time-base display can be calibrated as distance.

The B-scan technique basically combines a series of A-scans taken over a range of angles, to form a two-dimensional picture. As before, each A-scan corresponds to a single ultrasound pulse being emitted by the transducer and producing a series of reflected pulses from boundaries within the body. The ultrasound probe consists either of one crystal which can be moved rapidly through an arc or a series of crystals, each having a different orientation. The signals received from these probes are processed by a computer and displayed on a CRO. To make the combinations of these A-scans easy to interpret, the pulses are displayed as bright spots in the correct orientation of the A-scan. Consequently, the completed pattern of spots on the screen gives two-dimensional representation of the boundary positions in the body being scanned, which can 
then be photographed to give a permanent record. Problems can arise trying to move the transducer over the surface of the body without introducing air between it and skin. Coating the body with oilbased jelly has proved successful in overcoming these difficulties.

The main advantage of ultrasonic scanning is that the health risk factor for to human patients, and to those operating the system, is considered to be much less than in X-ray diagnosis. Other advantages are that the equipment is portable and relatively simple to use. Ultrasound systems have now been devised which can pick up and amplify the very small echoes produced by soft tissue boundaries within the body as well as those between hard and soft tissues. The information obtainable by the use of ultrasonic scanning is potentially far greater than for X-rays.

\section{X-RAYS:}

$\mathrm{X}$-rays are produced by bombarding metal targets with high speed electrons. They are electromagnetic radiation having very short wavelength. Their absorption by matter depends on the density and atomic weight of the material. They can pass through flesh surrounding the bones in human being but cannot pass through bones. This enables us to take an X-ray photograph of the bones of a human being, making it a valuable diagnostic tool. X-rays affect photographic plates in much the same way as visible light. A photographic plate, once exposed, will appear blackened on development. The degree of blackening is dependent on the total X-ray exposure. $X$-rays also produce fluorescence on the surface coated with zincsulphide or bariumplatinocynide. The process is similar to that by which visible light is produced on the screen of a CRO. The usefulness of $X$-rays is largely due to their penetrating power.

Radiographs or X-rays photographs are used for diagnosis and treatment of different diseases. Since $\mathrm{X}$-rays can damage healthy cells of the human body, great care is taken to avoid unnecessary exposure. In radiography the X-ray film is sandwiched between two screens which fluoresce when subjected to a small amount of X-radiation. The film is affected by the fluorescent light rather than by the X-rays, formerly X-rays were used to sensitize the film directly and much longer exposures were required.

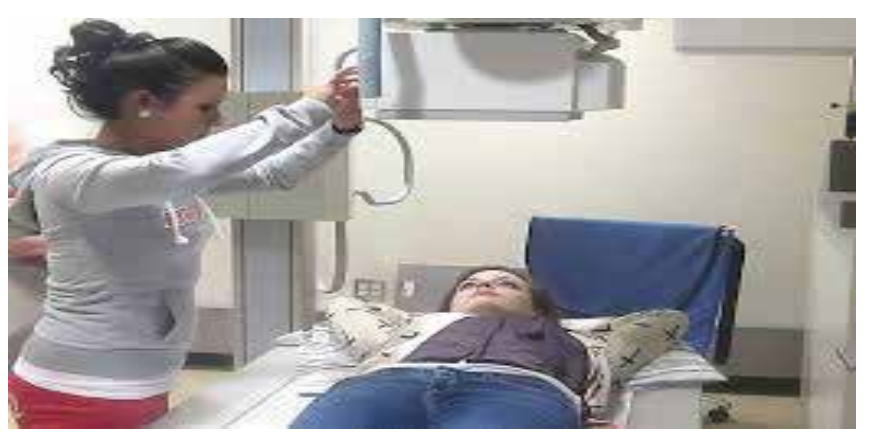

Fig(ii) X-rays Machine

Suspected bone fractures can be investigated since $\mathrm{X}$-rays of certain hardness can penetrate flesh but not bone. In the detection of lung tuberculosis by mass radiography use is made of the fact that diseased tissue is denser than healthy lung tissue which consists of air sacs and so the former absorbs $\mathrm{X}$-rays more strongly. When an organ is being $\mathrm{X}$-rayed whose absorptive power is similar to that of the surrounding tissue, a contrast medium is given to the patient, orally or by injection. This is less easily penetrated by the X-rays and enables a shadow of the organ to be obtained on a radiograph. $\mathrm{X}$-ray scanners enable three-dimensional analysis by imaging successive planes in the body. In the treatment of cancer by a radiotherapy, very hard (i.e. short wavelength) X-rays are used to destroy the cancer cells whose rapid multiplication causes malignant growth.

\section{LASERS:}

The full form of LASER is Light Amplification by Stimulated Emission of Radiation. It is a highly powerful, coherent, monochromatic source of light. Such light is of immense use in medicine, telecommunications, industries and holography. The lasers have such remarkable properties which have provided a number of possible applications in the different fields of pure science, medicine, biology, radar, communications, weaponry and micro-welding etc. Lasers have been used successfully in the treatment of detached retinas and show great promise in the treatment of cancer. Ophthalmologists additionally see the possibility of using the laser as an improved tool for vision research, and microsurgery. 


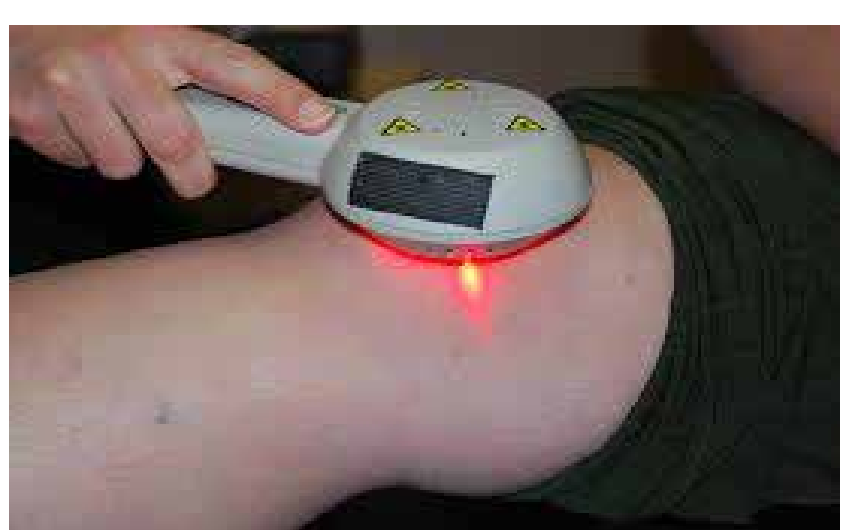

Fig(iii) Laser Theraphy

Detached retinas are developed by thousands of persons. This occurs when the fluid, vitreous humor, in the eye-ball seeps through a hole in the retina. This condition may eventually cause complete detachment of the retina from the back of the eyeball and blindness follows. Until now retina flaws have been treated by surgery, diathermy and by xenon flash tube, which use the same method as the laser beams, but in a considerably longer time. The laser flash, lasting a thousandth of a second, is much shorter than xenon flash retinal welding, which takes one half to one second to complete. This light can be precisely aimed and rarely requires more than a single pulse to attain action in the right location.

Microsurgery, other than in ophthalmology is an area of great potential use of laser light in medicine and its possibilities are being explored. A lot of work is now under way and is directed towards using an attenuated laser beam. Using much the same technique, experiments are going to be performed in an effort to use the laser to effect chromosomal changes. Laser beam can also be used in treatment of cancer. In 1963 Thufts- New England Medical Centre reported the preliminary success in the treatment of both human and animal cancers that had been transplanted into animals. An induced cancer of animal origin was also treated.

\section{ELECTROCARDIOGRAM (ECG):}

Electrocardiograph is a sensitive instrument that records the voltage and current waveforms associated with the action of the heart. The trace obtained is known as electrocardiogram or electroncardiogram. It is the photographic record of electrical variations that occur during contraction of muscles of heart. It is the manifestation of the operation of proteins and is used for the diagnosis of heart and neurological diseases. The beating of the heart and function of the brain require such kind of protein that produces electrical signals in cell. Biophysicists have also learned how these proteins generate their electrical signals and this knowledge has given us many therapies ranging from drug to treat abnormal heart beats to the treatment of epilepsy.

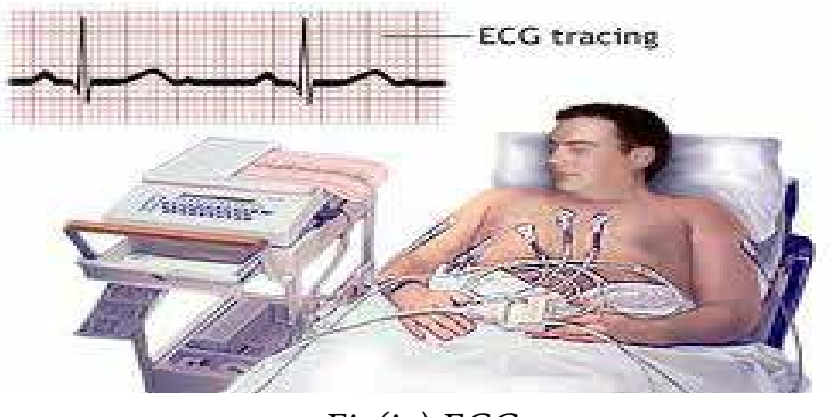

Fig(iv) ECG

Electrocardiogram is a graphic record of the electrical variations produced by the beating of the heart. This instrument was discovered by Einthoven, Father of Electrocardiography, in 1903. Using this device, the electrical activity of the heart can be detected by electrodes placed at the surface of the body. Its amplification can be displayed on a cathode ray tube, or for a permanent record, on a piece of graph paper. Electrocardiograms give information about the way the heart is beating. Analysis of such electrocardiograms also enables to analyze the extent of heart damage after a heart attack.

\section{ELECTROENCEPHALOGRAM (EEG):}

Electroencephalograph is a sensitive instrument that records the voltage waveforms associated with the rhythmical electric currents that pass through the brain. The trace obtained is known as an electroencephalogram. It represents a record of electrical activity generated in the brain. It is obtained through electrodes applied on the scalp (facial skin) or through microelectrodes placed within the brain tissue. It provides a physiologic assessment of cerebral activity. It measures voltage fluctuations resulting from ionic current flows within the neurons of the brain. 
In clinical contexts; EEG refers to the recording of the brain's spontaneous electrical activity over a short period of time as recorded from multiple electrodes placed on the scalp. Electrodes are applied on the scalp to record the electrical activity in various regions of the brain. The amplified activity of the neurons between any two of these electrodes is recorded on continuously moving paper; this record is called the encephalogram.

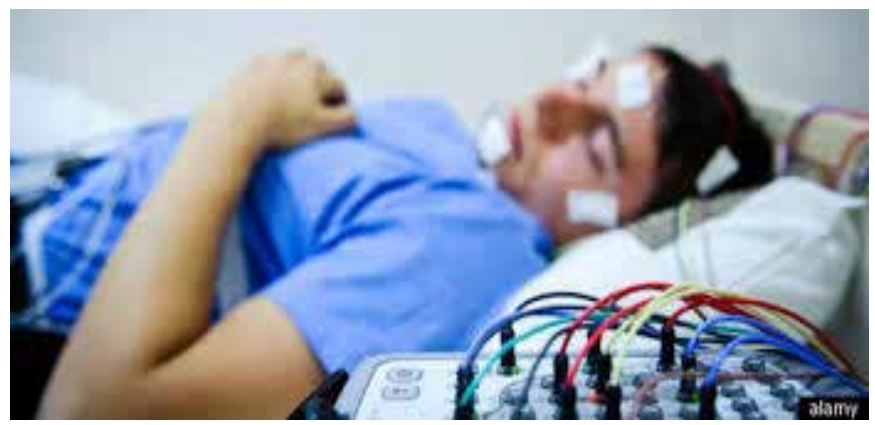

$\operatorname{Fig}(v) E E G$

It is described as one of the most surprising, remarkable and momentous developments in the history of clinical neurology. It is a useful test for diagnosing and evaluating seizure disorder, coma, encephalopathy or organic brain syndrome. Tumors, brain disorders, blood clots and infection may cause abnormal pattern in electrical activity. It is also used in making a determination of brain death.

\section{MAGNETIC RESONANCE IMAGING (MRI):}

Resonance is the phenomenon in which a large increase in the amplitude of a physical system occurs when it is acted on by an external driving force that oscillates at or near a particular frequency called the resonant frequency of the system. Many atomic nuclei behave as if they possess a spin. Such nuclei have an odd number of protons and/or an odd number of neutrons. Their spin causes such atoms to behave as tiny magnets (the moving charge in the nucleus creates a magnetic field). If an external magnetic field is applied to the atoms, they will line up in the field. However this alignment is not perfect and the nuclei rotate about the direction of the magnetic field. This type of motion is known as precession and the frequency of precession is called Larmor frequency which depends on the nature of the nucleus and the strength of the magnetic field.
The Larmor frequency is found to lie in the radio frequency region of the electromagnetic spectrum.

If a short pulse of radio waves of frequency equal to that of the Larmor frequency is applied, the atoms will resonate and, when the pulse ends, the atoms will return to their equilibrium state. In this process, radio frequency radiation will be emitted. The time taken between the end of a pulse and the emission of the radio frequency radiation forms the basis of magnetic resonance imaging (MRI).

The subject of the investigation is placed between the poles of a very large magnet which produces a uniform magnetic field in excess of one tesla. Since hydrogen atoms are in great abundance in body tissue, hydrogen nuclei are used for imaging. All these nuclei within the uniform magnetic field would have the same Larmor frequency. In order to locate the position within the patient of particular hydrogen atoms, a non-uniform but accurately calibrated magnetic field is also applied. This provides a unique value for the magnetic field at any point and coupled with the particular value of the Larmor frequency enables the hydrogen nuclei to be located.

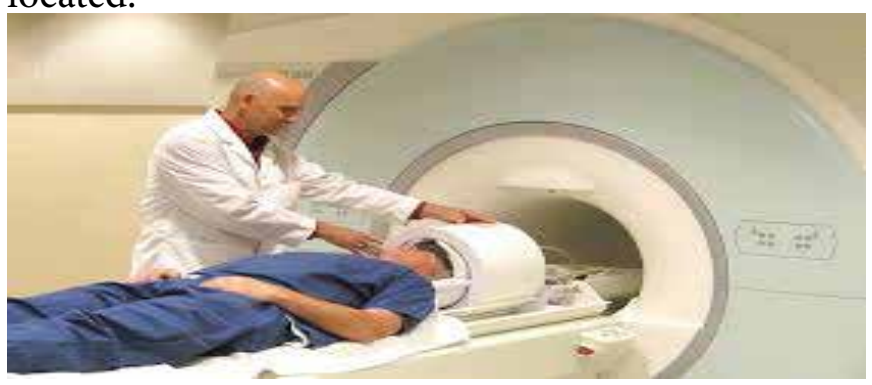

Fig(vi) $M R I$

Radio frequency pulses are transmitted to the patient by means of suitable coils. These coils are also used to detect the radio frequency emissions from the patient. The received signals are processed in order to construct an image. Modern developments have allowed for the further refinement of the information available so that not only can detailed images of body structures be obtained but also "Image flow" images showing only arteries and veins have been created.

MRI can be used for the brain but in the special case of new-born babies, the use of MRI can cause 
problems. It requires the child to remain very still during its technically complex and long assessment procedures. The outcome is likely to provide only intermittent and not continuousinformation on brain metabolism. Also, MRI does not measure directly the supply of oxygen to the child's brain tissues.

\section{HOLOGRAM:}

It is an intermediate photographic record which contains information for reproducing a three dimensional image by holography. Holography is a technique of reproducing three dimensional images without cameras or lenses. The object to be photographed is illuminated with laser light; the light reflected from the object is combined with direct light from the source to produce an interference pattern on a photographic plate. The three dimensional image can be viewed by illuminating the interference pattern obtained above with the original light, that is usually a laser beam. Unlike the conventional two dimensional photographs, this image cannot be seen without the original light.

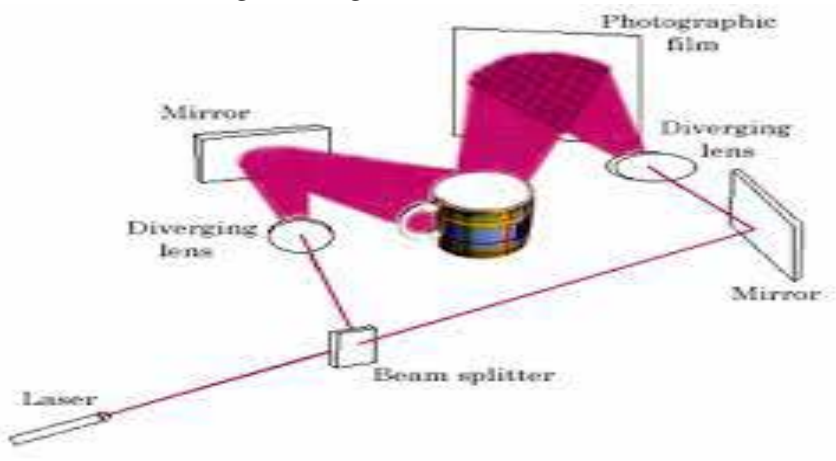

Fig(vii) Hologram machine

Hologram is a three - dimensional image recorded on a special photographic plate by light from a laser. When developed and illuminated the image not only looks real but it appears to float in space and to move when the viewer moves as with a real object. In medicine a hologram of a living brain can reveal to surgeons the exact size and location of a tumor. It is also useful in the design of artificial joints.

\section{COMPUTED TOMOGRAPHY (CT) SCANNING:}

The aim of CT scanning is to make an image of a section through the body from measurements made about its axis. The section through the body is divided up into a series of small units called voxels.
The image of each voxel would have a particular intensity known as a pixel. The pixels are built up from measurements of X-ray intensity made along a series of different directions around the section of the body.

If a beam of X-rays is directed from the left, then detectors will give readings depending upon the number on each voxel representing the pixel intensity. This allows the reconstruction of four voxels. The X-ray tube and detectors are now rotated through $45^{\circ}$ and new detector readings are found. These new detector readings are added to the readings already obtained for the voxels. The procedure is repeated after rotating the X-ray tube and the detectors through a further $45^{\circ}$. The final images are taken after rotating the X-ray tube and the detectors through a further $45^{\circ}$.

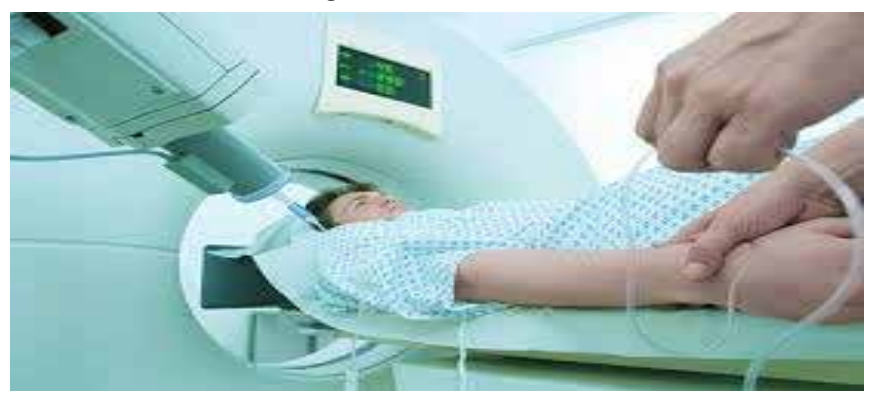

Fig(viii) CT Scanning

In order to obtain the original pattern of pixels, two operations must be performed.

1. The background intensity must be removed. The background intensity is the total of each set of detector readings.

2. After deduction of the background, the result must be divided by three to allow for the duplication of the views of the section.

The pattern of pixels for the section now emerges. In practice, the image of each section is built up from many small pixels, each viewed from many different angles. The collection of the data and its construction into a display on a screen requires a powerful computer and complicated programmes. In fact, the reconstruction of each pixel intensity value requires more than one million computations. The contrast and brightness of the image of the section as viewed on the TV screen can be varied to achieve optimum results. In order to build up an image of the whole 
body, the procedure would be repeated for further sections through the body. All the data for all the sections can be stored in the computer memory to create a three-dimensional image. Views of the body from different angles may be constructed.

\section{POSITRON EMISSION TOMOGRAPHY (PET):}

Electron has its antiparticle called positron which is identical to electron in mass and spin but has opposite charge. When an electron and a positron are together they disappear and give energy. This phenomenon is called pair annihilation. The opposite i.e. formation of a pair of particle and antiparticle is also possible from energy which is called pair creation. So, positron can also be emitted along with the electron with the help of incident radiation because pair annihilation and pair production are the perfect examples of mass energy equivalence.

PET is applied to understand the functioning of human brain by using high resolution scanning techniques. PET scanning involves the emission of particles of antimatter by compound ejected into the body being scanned. These particles, positrons, are neutralized by their opposites, electrons, and energy is released in the form of radiation due to annihilation of matter. Detectors arranged around the body pick up the energy released and use it to follow the movements of the injected compound and its metabolism. These relatively new radiological techniques provide much safer means of examining internal body structures. These techniques are sensitive to local brain metabolism or even to local concentration of complex psychoactive biochemical molecules that are thought to regulate brain function and for some forms of drug addiction.

\section{CONCLUSION:}

All the above discussions present a few of the major advances in the field of medical science which utilizes the laws of physics. It also presents the impacts of physics on other sciences including medical physics and describes some of the non-invasive techniques of medical science. For many years, physicists have been collaborating with doctors, physicians, chemists, pharmacologists and computer scientists in a highly successful effort to solve some of society's most pernicious health problems. Their efforts have established Ultrasound, X-rays, Lasers, ECG, EEG, MRI, Hologram, CT Scanning and PET as a standard part of modern medical practice which would be impossible without the knowledge of physics. A series of advances in Physics has a decisive and direct impact on medical science. Physics is addressing increasingly complex problems of medical science. So, the role of physics in medical science can hardly be exaggerated and the future of medical science lies in the development of physics.

\section{REFERENCES:}

[1] Aman Rao, 1989, Dictionary of Physics, Anmol Publications, New Delhi, India.

[2] Lamichhane H., 2011, What is EEG? Resolution, vol. 1.

[3] Lamsal M. R. et. al., 2009, Themes of Physics, Koselee Prakashan, Nepal.

[4] Poudel P. R., 2011, Physics in Medical Science, The Himalayan Physics, vol. 2.

[5] Saxena, Gupta and Saxena, 1990, Fundamentals of Solid State Physics, Pragati Prakashan Meerut, India

[6] Tom Duncan, 1987, Advanced Physics $4^{\text {th }}$ Edition, John Murray Publishers Ltd., London.

[7] University of Cambridge, 1995, A Level Science Applications Support Booklet.

[8] http/www.google.com 